International Journal of Pure and Applied Mathematics

Volume 94 No. 4 2014, 525-532

ISSN: 1311-8080 (printed version); ISSN: 1314-3395 (on-line version)

url: http://www.ijpam.eu

doi: http://dx.doi.org/10.12732/ijpam.v94i4.7

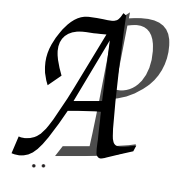

ijpam.eu

\title{
SOME PROBLEMS FOR FRACTIONAL ANALOGUE OF LAPLACE EQUATION
}

\author{
B.Kh. Turmetov ${ }^{1}$, B.T. Torebek ${ }^{2}$, Sh. Ontuganova ${ }^{3}$ \\ 1,2,3 Akhmet Yasawi International Kazakh-Turkish University \\ Sattarkhanov Street 29, 161200, Turkistan, KAZAKHSTAN
}

\begin{abstract}
In this paper we study some boundary value problems for fractional analogue of Laplace equation in a rectangular. Theorems about existence and uniqueness of a solution of the considered problems are proved by spectral method.
\end{abstract}

AMS Subject Classification: 34A08, 35R11, 74S25

Key Words: fractional Laplace equation, Caputo operator, boundary value problems

\section{Introduction}

Let's introduce definition of operators of fractional integral - differen-tiation. Let a function $f(t)$ be given in a interval $[0, \ell], \ell<\infty$. For any $\alpha>0$ operator of fractional integration of $\alpha$ order in Riemann - Liouville sense is the expression:

$$
I^{\alpha}[f](t)=\frac{1}{\Gamma(\alpha)} \int_{0}^{t}(t-s)^{\alpha-1} f(s) d s .
$$

Let $m-1<\alpha \leq m, m=1,2, \ldots$. Then the operator

Received: March 16, 2014

(c) 2014 Academic Publications, Ltd. url: www.acadpubl.eu

${ }^{\S}$ Correspondence author 


$$
{ }_{R L} D^{\alpha}[f](t)=\frac{d^{m}}{d t^{m}} I^{m-\alpha}[f](t)
$$

is called derivative of $\alpha$ order in Riemann - Liouville sense, and the operator

$$
{ }_{C} D^{\alpha}[f](t)={ }_{R L} D^{\alpha}\left[f(t)-f(0)-\ldots-\frac{f^{(m-1)}(0)}{(m-1) !} t^{m-1}\right]
$$

is a derivative of $\alpha$ order in Caputo sense [1]. If $f(t) \in C^{m}[0, l]$, then the operator ${ }_{C} D^{\alpha}$ can be reduced to the form [1]:

$$
{ }_{C} D^{\alpha}[f](t)=I^{m-\alpha}\left[f^{(m)}\right](t) .
$$

Furthermore, we will use another form of the operator of fractional order, namely sequential derivative of $k \alpha, k=1,2, \ldots$ order, i.e. expression[2]:

$$
D^{\alpha}={ }_{C} D^{\alpha}, 0<\alpha \leq 1, D^{k \alpha}=D^{\alpha} D^{(k-1) \alpha}, k=2,3, \ldots
$$

In domain $\Omega=\left\{(x, y) \in R^{2}: 0<x<1,0<y<1\right\}$ consider the following equation:

$$
D_{x}^{2 \alpha} u(x, y)+u_{y y}(x, y)=0,(x, y) \in \Omega, \frac{1}{2}<\alpha \leq 1 .
$$

Here $D_{x}^{2 \alpha}$ means $D_{x}^{2 \alpha}=D_{x}^{\alpha} D_{x}^{\alpha}$ and the operator $D_{x}^{\alpha}$ acts by a variable $x$.

Regular solution of the equation (1.1) is a function $u \in C(\bar{\Omega})$, such that $D_{x}^{\alpha} u, D_{x}^{2 \alpha} u, u_{y y} \in C(\Omega)$. Since when $\alpha=1$ equation ${ }_{C} D^{1}=\frac{d}{d y}$ holds, then in this case $D_{x}^{2}+\frac{\partial^{2}}{\partial y^{2}}=\Delta$, i.e. equation (1.1) coincides with the usual Laplace equation.

\section{Formulation of the Problem}

In the domain $\Omega$ we consider the following problems:

Problem D. Find a regular solution of the equation (1.1), satisfying the fol-lowing boundary conditions:

$$
\begin{gathered}
u(0, y)=f(y), u(1, y)=g(y), 0 \leq y \leq 1, \\
u(x, 0)=0, u(x, 1)=0,0 \leq x \leq 1 .
\end{gathered}
$$

Problem P. Find a regular solution of the equation (1.1), such that $u_{y}(x, y) \in$ $C(\bar{\Omega})$, satisfying the boundary conditions $(2.1)$ and

$$
u(x, 0)=u(x, 1), u_{y}(x, 0)=u_{y}(x, 1), 0 \leq x \leq 1 .
$$


Problem N. Find a regular solution of the equation (1.1), such that $u_{y}(x, y) \in C(\bar{\Omega})$, satisfying the boundary conditions $(2.1)$ and

$$
u_{y}(x, 0)=0, u_{y}(x, 1)=0,0 \leq x \leq 1 .
$$

Note, that Dirichlet type problem for fractional analogue of the Laplace equation:

$$
{ }_{C} D_{x}^{\alpha} u(x, y)+u_{y y}(x, y)=0,(x, y) \in \Omega, 1<\alpha<2 .
$$

has been studied in [3]. Since for the operator of fractional differentiation in Caputo sense, in general, equality (see [2])

$$
{ }_{C} D^{\alpha}{ }_{C} D^{\beta} \neq{ }_{C} D^{\alpha+\beta}, 0<\alpha, \beta \notin N,
$$

holds, then the considered problem D differs from the Dirichlet problem for the equation (2.5). Necessary of research boundary value problems for equation (1.1) is determined by using fractal Laplace equation to describe the production processes in mathematical modeling of socio-economic systems [4]. In [4] attention is drawn to the fact that the problem of finding a generalized two-factor Cobbe-Douglas function is reduced to a Dirichlet problem for the generalized Laplace equation of fractional order.

\section{Solution of One-Dimensional Equation with Fractional Derivative}

For further research, we need to give some information about solutions of differential equations of the form:

$$
D^{2 \alpha}[y](t)-\mu^{2} y(t)=0, \mu>0 .
$$

We will look for a solution of the equation (3.1) in the class of functions $y(t), D^{\alpha} y(t) \in C[0,1], D^{2 \alpha} y(t) \in C(0,1)$. Since $\mu>0$, then the equation (3.1) can be rewritten in the form:

$$
\left(D^{\alpha}-\mu\right)\left(D^{\alpha}+\mu\right) y(t)=0 .
$$

We construct the general solution of the equation (3.2). It is known (see [1]) that a particular solution of the equation $\left(D^{\alpha}-\mu\right) y(t)=0$ is a function $y(t)=$ $E_{\alpha, 1}\left(\mu t^{\alpha}\right)$, where $E_{\alpha, \beta}(z)=\sum_{k=0}^{\infty} \frac{z^{k}}{\Gamma(\alpha k+\beta)}$ - the Mittag - Leffler type function [1]. Then the functions

$$
\left\{E_{\alpha, 1}\left(\mu t^{\alpha}\right), E_{\alpha, 1}\left(-\mu t^{\alpha}\right)\right\}
$$


are solutions of the equation (3.2). It is easy to show that the functions $E_{\alpha, 1}\left(\mu t^{\alpha}\right)$ and $E_{\alpha, 1}\left(-\mu t^{\alpha}\right)$ are linearly independent. Hence, the system of the functions (3.3) are the fundamental solutions of the equation (3.1), and, therefore, the general solution of this equation has the form:

$$
y(t)=C_{1} E_{\alpha, 1}\left(-\mu t^{\alpha}\right)+C_{2} E_{\alpha, 1}\left(\mu t^{\alpha}\right) .
$$

\section{Research the Problem D}

Application of the Fourier method to solve the problem D leads to a spectral problem

$$
Y^{\prime \prime}(y)+\lambda Y(y)=0,0<y<1, Y(0)=0, Y(1)=0 .
$$

Eigen values of the problem have the form $\lambda_{k}=(\pi k)^{2}, k=1,2, \ldots$, and corresponding their eigen functions $Y_{k}(y)=\sqrt{2} \sin k \pi y$ form orthonormal basis in the space $L_{2}(0,1)$. Thus, for all $y$ any regular solution of the problem $\mathrm{D}$ can be represented as the following series:

$$
u(x, y)=\sum_{k=1}^{\infty} u_{k}(x) Y_{k}(y)
$$

It is known, that if functions $f(y), g(y)$ are sufficiently smooth in $[0,1]$ and satisfy conditions $f(0)=f(1)=0, g(0)=g(1)=0$, then they are uniquely represented in the form of a uniformly and absolutely convergent Fourier series in the system $Y_{k}(y)$

$$
f(y)=\sum_{k=1}^{\infty} f_{k} Y_{k}(y), g(y)=\sum_{k=1}^{\infty} g_{k} Y_{k}(y),
$$

where $f_{k}=\int_{0}^{1} f(y) Y_{k}(y) d x, g_{k}=\int_{0}^{1} g(y) Y_{k}(y)$. Putting (4.1) into the equation (1.1) and boundary value condition (2.2), to find unknown functions $u_{k}(x)$ we get the following problem:

$$
\begin{gathered}
D_{y}^{2 \alpha} u_{k}(x)-\lambda_{k} u_{k}(x)=0,0<x<1, \\
u_{k}(0)=f_{k}, u_{k}(1)=g_{k} .
\end{gathered}
$$

Due to the equality (3.4) general solution of the equation (4.2) has the form:

$$
u_{k}(x)=C_{1} E_{\alpha, 1}\left(-k \pi x^{\alpha}\right)+C_{2} E_{\alpha, 1}\left(k \pi x^{\alpha}\right) .
$$


Putting the function (4.4) into the boundary condition (4.3), we obtain

$$
u_{k}(x)=C_{k}(x) f_{k}+S_{k}(x) g_{k},
$$

where

$$
\begin{gathered}
C_{k}(x)=\frac{E_{\alpha, 1}\left(\sqrt{\lambda_{k}}\right) E_{\alpha, 1}\left(-\sqrt{\lambda_{k}} x^{\alpha}\right)-E_{\alpha, 1}\left(\sqrt{\lambda_{k}} x^{\alpha}\right) E_{\alpha, 1}\left(-\sqrt{\lambda_{k}}\right)}{2 \sqrt{\lambda_{k}} E_{2 \alpha, \alpha+1}\left(\lambda_{k}\right)}, \\
S_{k}(x)=\frac{x^{\alpha} E_{2 \alpha, \alpha+1}\left(\lambda_{k} x^{2 \alpha}\right)}{E_{2 \alpha, \alpha+1}\left(\lambda_{k}\right)} .
\end{gathered}
$$

It is easy observe, that the function $E_{\alpha, 1}\left(\lambda_{k} x^{\alpha}\right)$ satisfies equation:

$$
y^{\prime \prime}(x)-\lambda_{k R L} D^{2-\alpha} y(x)=0,0<x<1 .
$$

Moreover, it's known that (see [5]), a regular non-constant solution of the equation (4.7) (function $y(x)$ belongs to the class $C[0,1] \cap C^{2}(0,1)$ ) can not attain its positive maximum (negative minimum) within the segment. It's easy to show, that functions $C_{k}(x)$ and $S_{k}(x)$ are solutions of the equation (4.7) and

$$
\begin{aligned}
& C_{k}(0)=1, \quad C_{k}(1)=0, \\
& S_{k}(0)=0, \quad S_{k}(1)=1 .
\end{aligned}
$$

Consequently, $0 \leq S_{k}(x), C_{k}(x) \leq 1$, for all $x \in[0,1]$. Further, if the function $\varphi(x)$ belongs to the class $C^{m+\varepsilon}[0, l], m=0,1, \ldots, 0<\varepsilon<1$, then for the Fourier coefficients of this function we have the following estimate (see [6]):

$$
\left|\varphi_{k}\right|=O\left(\frac{1}{k^{m+\varepsilon}}\right), k \rightarrow \infty .
$$

If $f^{\prime \prime}(y) \in C^{\varepsilon}[0,1], g^{\prime}(y) \in C^{\varepsilon}[0,1]$ and conditions $f(0)=f(1)=g(0)=$ $g(1)=0$ hold, then

$$
\left|f_{k}\right| \leq \frac{C}{k^{2+\varepsilon}},\left|g_{k}\right| \leq \frac{C}{k^{1+\varepsilon}}, C-\text { const } .
$$

For these functions we receive:

$$
\left|u_{k}(x)\right| \leq C\left(\frac{1}{k^{2+\varepsilon}}+\frac{1}{k^{1+\varepsilon}}\right) .
$$

Then the series (4.1) converges uniformly in the region $\bar{\Omega}$, hence its sum is $u(x, y) \in C(\bar{\Omega})$. Further, for the function $E_{\alpha, \beta}(z)$ as $|z| \rightarrow \infty$ we get asymptotical estimate $[2]$ :

$$
E_{\alpha, \beta}(z)=\frac{1}{\alpha} z^{\frac{(1-\beta)}{\alpha}} e^{z^{\frac{1}{\alpha}}}-\sum_{k=1}^{p} \frac{z^{-k}}{\Gamma(\beta-\alpha k)}+O\left(\frac{1}{|z|^{p+1}}\right)
$$


where $|\arg z| \leq \rho_{1} \pi, \rho_{1} \in\left(\frac{\alpha}{2}, \min \{1, \alpha\}\right), \alpha \in(0,2)$. If $\arg z=\pi$, then $E_{\alpha, \beta}(z)=$ $\frac{1}{1+|z|},|z| \rightarrow \infty$. Using these estimations, we obtain

$$
\begin{gathered}
2 x^{\alpha} \sqrt{\lambda_{k}} E_{2 \alpha, \alpha+1}\left(\lambda_{k} x^{2 \alpha}\right)=\frac{x^{\alpha}}{\alpha} e^{\lambda_{k}^{\frac{1}{2 \alpha}} x}+O\left(\frac{1}{\lambda_{k}}\right), \\
E_{\alpha, 1}\left(\sqrt{\lambda_{k}} x^{\alpha}\right)=\frac{1}{\alpha} e^{\lambda_{k}^{\frac{1}{2 \alpha}} x}+O\left(\frac{1}{\sqrt{\lambda_{k}}}\right), \\
E_{\alpha, 1}\left(-\sqrt{\lambda_{k}} x^{\alpha}\right)=O\left(\frac{1}{\sqrt{\lambda_{k}}}\right), x \geq x_{0} .
\end{gathered}
$$

Then

$$
S_{k}(x)=O\left(e^{\lambda_{k}^{\frac{1}{2 \alpha}}(x-1)}\right), C_{k}(x)=O\left(\frac{1}{\sqrt{\lambda_{k}}}\right) .
$$

Term by term taking differentiation from the series (4.1) twice by $y$, we have:

$$
u_{y y}(x, y)=-\sum_{k=1}^{\infty} \lambda_{k} u_{k}(x) Y_{k}(y)
$$

Then for all $x \geq x_{0}>0,0 \leq y \leq 1$ we get

$$
\left|u_{y y}(x, y)\right| \leq \sum_{k=1}^{\infty}\left|\lambda_{k}\right|\left|u_{k}(x)\right| \leq C \sum_{k=1}^{\infty}\left[\frac{e^{-k \pi(1-x)}}{k^{\varepsilon}}+\frac{1}{k^{\varepsilon+1}}\right]<\infty .
$$

Analogously we estimate the series: $D^{2 \alpha} u(x, y)=\sum_{k=1}^{\infty} \lambda_{k} u_{k}(x) Y_{k}(y)$. Then $u_{y y}(x, y), D^{2 \alpha} u(x, y) \in C(\Omega)$. Uniqueness of a solution of the problem $\mathrm{D}$ follows from uniqueness of solution of the problems (4.2), (4.3). Thus, we have proved the following proposition.

Theorem 1. Let $\frac{1}{2}<\alpha \leq 1, f(y) \in C^{2+\varepsilon}[0,1], g(y) \in C^{1+\varepsilon}[0,1]$ and conditions $f(0)=f(1)=0, g(0)=g(1)=0$ hold. Then solution of the problem $D$ exists, it is unique and represented as follows:

$$
u(x, y)=\sum_{k=1}^{\infty}\left[f_{k} C_{k}(x)+g_{k} S_{k}(x)\right] \sin k \pi y
$$

where $f_{k}, g_{k}$ - Fourier coefficients of functions $f(y), g(y)$, moreover, $C_{k}(x)$ and $S_{k}(x)$ are defined by equalities (4.6) and (4.7), respectively. 


\section{Research the Problem $P$}

In the case of boundary conditions (2.3) of the problem $\mathrm{P}$, the corresponding spectral problem has the form

$$
-Y^{\prime \prime}(y)=\lambda Y(y), 0<y<1, Y(0)=Y(1), Y^{\prime}(0)=Y^{\prime}(1) .
$$

Eigen values of this problem are $\lambda_{k}=(2 k \pi)^{2}, k=0,1, \ldots$, and corresponding their eigen functions have the form:

$$
\left\{\begin{array}{l}
Y_{0}(y)=1, Y_{k, 1}(y)=\sqrt{2} \cos 2 k \pi y \\
Y_{k, 2}(y)=\sqrt{2} \sin 2 k \pi y, k=1,2, \ldots
\end{array}\right.
$$

System of the functions (5.1) form orthonormal basis in the space $L_{2}(0,1)$. Then repeating the procedure of paragraph 4 , we obtain

Theorem 2. Let $\frac{1}{2}<\alpha \leq 1, f(y) \in C^{3+\varepsilon}[0,1], g(y) \in C^{2+\varepsilon}[0,1]$ and conditions $f(0)=f(1), f^{\prime}(0)=f^{\prime}(1), g(0)=g(1), g^{\prime}(0)=g^{\prime}(1)$ hold. Then solution of the problem $P$ exists, it is unique and represented as follows:

$$
\begin{gathered}
u(x, y)=\left(1-x^{\alpha}\right) f_{0}+\sum_{k=1}^{\infty}\left[f_{2 k} C_{k}(x)+g_{2 k} S_{k}(x)\right] \cos 2 k \pi x+ \\
+x^{\alpha} g_{0}+\sum_{k=1}^{\infty}\left[f_{2 k-1} C_{k}(x)+g_{2 k-1} S_{k}(x)\right] \sin 2 k \pi y,
\end{gathered}
$$

where $f_{k}, g_{k}$ - Fourier coefficients of the functions $f(y)$ and $g(y)$ by the system (5.1), $C_{k}(x)$ and $S_{k}(x)$ are defined by equalities (4.5) and (4.6), respectively.

\section{Research the Problem N}

In the case of the problem $\mathrm{N}$ the corresponding spectral problem has the form:

$$
-Y^{\prime \prime}(y)=\lambda Y(y), 0<y<1, Y^{\prime}(0)=Y^{\prime}(1)=0 .
$$

Eigen values of this problem have the form $\lambda_{k}=(2 k \pi)^{2}, k=0,1, \ldots$, and eigen functions $Y_{0}(y)=1, Y_{k}(y)=\sqrt{2} \cos k \pi y, k=1,2, \ldots$, forms orthonormal basis in the space $L_{2}(0,1)$. Then, as in the case of the problem $\mathrm{D}$ and problem $\mathrm{P}$, for the problem $\mathrm{N}$ we obtain the following proposition. 
Theorem 3. Let $\frac{1}{2}<\alpha \leq 1, f(y) \in C^{3+\varepsilon}[0,1], g(y) \in C^{2+\varepsilon}[0,1]$ and conditions $f^{\prime}(0)=f^{\prime}(1)=0, g^{\prime}(0)=g^{\prime}(1)=0$ hold. Then solution of the problem $N$ exists, it is unique and represented as follows:

$$
u(x, y)=\left(1-x^{\alpha}\right) f_{0}+x^{\alpha} g_{0}+\sum_{k=1}^{\infty}\left[f_{k} C_{k}(x)+g_{k} S_{k}(x)\right] \cos k \pi x,
$$

where $f_{k}, g_{k}$ - Fourier coefficients of the functions $f(y), g(y)$, by the system $Y_{k}(y), k=0,1, \ldots, C_{k}(x)$ and $S_{k}(x)$ are defined by equalities (4.5) and (4.6), respectively.

\section{Acknowledgements}

This paper is financially supported by the grant of the Ministry of Science and Education of the Republic of Kazakhstan (Grant No. 0830/GF2).

\section{References}

[1] A.A.Kilbas, H.M.Srivastava, J.J.Trujillo Theory and Applications of Fractional Differential Equations. Elsevier. North-Holland. Mathematics studies. -539p. (2006)

[2] I.Podlubny Fractional Differential equations. Mathematics in Scince and Engineering. V.198. Academic Press. -356 p. (1999)

[3] O.Kh.Masaeva Dirichlet Problem for the Generalized Laplace Equation with the Caputo Deriva-tive. Differential Equations. V.48. 3. 449-454. (2012)

[4] A.M.Nakhushev On Mathematical and Information Technologies for Modeling and Control of Regional Development. Dokl. Adygsk. (Cherkessk.) Mezhdunar. Akad. Nauk V.9. 1. 128-137.(2007) (In Russian)

[5] A.M.Nakhushev Fractional Calculus and Its Applications. Fizmatlit, Moscow, (2003) (In Russian)

[6] V.A.Il'in, E.G.Poznyak Foundations of Mathematical Analysis. Fizmatlit, Moscow, -464p. (2002) (In Russian). 